\title{
Tradução e Validação de Algoritmo para Identificação de Lesão Hepática Induzida por Medicamentos
}

\author{
Michele John Müller Lunardelli, ${ }^{1}$ Matheus William Becker, ${ }^{1}$ Carine Raquel Blatt ${ }^{2}$
}

\begin{abstract}
RESUMO
Introdução: A lesão hepática induzida por medicamentos é um tipo de reação adversa a medicamento (RAM), cuja ocorrência é a causa de falha hepática fulminante em $13 \%$ a $30 \%$ dos casos. O Russel Uclaf Causality Assessment Method (Rucam) é um algoritmo específico para a determinação de lesão hepática induzida por medicamentos. O objetivo deste trabalho é traduzir e validar o algoritmo Rucam para o português. Método: Foi realizada a tradução e tradução inversa do algoritmo. A validação de conteúdo foi verificada pela aplicação do algoritmo traduzido a um caso real, por um grupo de médicos, farmacêuticos e enfermeiros. Resultados: Foram obtidas 41 aplicações do algoritmo. 0 tempo médio de aplicação foi de $17 \pm 10$ minutos. O escore médio foi de 7,58 +3,48 (provável relação de causalidade). As diferenças do escore para as diferentes categorias profissionais não foi estatisticamente significativa $(p=0,800)$. Discussão: $O$ algoritmo Rucam possui sete domínios: tempo de início da doença hepática, duração da doença, fatores de risco, uso concomitante de medicamentos, exclusão de causas alternativas, história prévia do medicamento suspeito e resposta à reexposição ao medicamento. Tais informações permitem maior acurária na confirmação da suspeita da RAM, favorecendo a identificação dos medicamentos com potencial hepatotóxico. Conclusão: O algoritmo Rucam, traduzido para o português, mostrou-se uma ferramenta útil para a investigação de causalidade entre a lesão hepática e medicamentos, que pode ser aplicado por médicos, enfermeiros e farmacêuticos.
\end{abstract}

Palavras-chave: Doença hepática crônica induzida por substâncias e drogas. Reação adversa ou efeito adverso relacionado a medicamentos. Falência hepática. Algoritmos.

\section{TRANSLATION AND VALIDATION FOR DRUG-INDUCED LIVER INJURY ALGORITHM}

\section{ABSTRACT}

Introduction: Drug induced liver injury is a type of adverse drug reaction. Its occurrence is the cause of fulminant liver failure in $13 \%$ to $30 \%$ of cases. The. Russel Uclaf Causality Assessment Method (Rucam) is a specific algorithm for the determination of drug-induced liver disease. The aim is to translate and validate the Rucam algorithm into the Portuguese. Methods: The translation and the inverse translation of the algorithm was performer. The validation of content verified by the application of the algorithm translated to a real case, by doctors, pharmacists and nurses. Results: 41 valid answers were obtain. The average time for the application was $17+10$ minutes. The mean score observed was $7.58+3.48$ (probable causal relationship). The score differences found between the different professional categories were not statistically significant $(p=0.800)$. Discussion: The Rucam algorithm has seven domains: time of onset of liver disease, duration of disease, risk factors, concomitant use of drugs, and exclusion of alternative causes, previous virus history, and response to drug exposure. Such information allows greater accuracy in the confirmation of suspected adverse reaction, favoring the identification of drugs with hepatotoxic potential. Conclusion: The Portuguese translated Rucam algorithm proved to be a useful tool for investigating causality between liver injury and drugs, which can be apply by doctors, nurses and pharmacists.

Keywords: Chemical and drug induced liver injury. Chronic. Drug-related side effects and adverse reactions. Liver Failure. Algorithms.

RECEBIDO EM: 21/2/2020

MODIFICAÇÕES SOLICITADAS EM: 28/4/2020

ACEITO EM: 3/5/2020

\footnotetext{
Universidade Federal de Ciências da Saúde de Porto Alegre - UFCSPA. Porto Alegre/RS, Brasil.

Autora correspondente. Universidade Federal de Ciências da Saúde de Porto Alegre - UFCSPA. R. Sarmento Leite, 245, Centro Histórico - Porto Alegre/RS, Brasil. 90050-170. http://lattes.cnpq.br/4746842392238066. https://orcid.org/0000-0001-5935-1196. carineblatt@ufcspa.edu.br
} 


\section{INTRODUÇÃO}

A lesão hepática induzida por medicamentos ou hepatite medicamentosa, ou, ainda, Dili do inglês Drug induced liver injury - é um tipo de reação adversa a medicamento. Estudos apontam a ocorrência de Dili como sendo a causa de falha hepática fulminante em $13 \%$ a $30 \%$ dos casos em que sua ocorrência é confirmada (TESCHKE; ANDRADE, 2016; TESCHKE, 2019). Sua notificação, porém, ainda é muito baixa, razão pela qual os mecanismos de toxicidade hepática de alguns medicamentos ainda são desconhecidos. 0 motivo da subnotificação talvez esteja relacionado à dificuldade de identificação e diagnóstico, posto que este depende de um elevado grau de suspeição por parte dos profissionais de saúde e ampla investigação de outros diagnósticos diferenciais (TESCHKE; ANDRADE, 2016; DANAN; TESCHKE, 2019).

Alguns algoritmos de causalidade contribuem para dar maior consistência ao diagnóstico de reações adversas a medicamentos (TESCHKE; ANDRADE, 2016; TAN et al., 2018; DANAN; TESCHKE, 2016). A avaliação de causalidade tem como objetivo analisar a probabilidade de um determinado medicamento ser a causa de um efeito adverso, observado por meio da relação temporal entre a administração do medicamento e o aparecimento do evento (time to onset), da exclusão de outros possíveis diagnósticos, ou seja, causas alternativas que possam explicar o caso, da existência de fundamentos na literatura científica para o medicamento suspeito, da reação do organismo ante a retirada do medicamento (melhora nos sinais e sintomas na retirada do medicamento), bem como a piora nos sinais e sintomas com a reexposição ao medicamento (rechallenge) (TESCHKE; ANDRADE, 2016; TAN et al., 2018; DANAN; TESCHKE, 2016).

No Brasil, a maior parte dos estudos de farmacovigilância utiliza-se do algoritmo de Naranjo para estabelecer a relação de causalidade (BLATT; BECKER; LUNARDELLI, 2016; BECKER et al., 2019a). Tal algoritmo foi desenvolvido por Naranjo et al. (1981) no âmbito da pesquisa clínica, e publicado em 1981, e é composto por dez perguntas, cujas respostas são objetivas e diretas (sim ou não) (NARANJO et al., 1981). Estudo aponta que o algoritmo de Naranjo, apesar de amplamente utilizado, não é a melhor opção para a prática clínica diária, pois apresenta baixa sensibilidade e valor preditivo negativo, bem como uma capacidade limitada de distinguir entre as categorias adjacentes de probabilidade como possível ou provável, por exemplo (MILKKOVIC; DOBRIC; DRAGOJEVIC-SIMIC, 2012).
Existem alguns algoritmos específicos para a identificação da causalidade entre a ocorrência de lesão hepática e o uso de medicamento, tais como Russel Uclaf Causality Assessment Method (Rucam) (DANAN; TESCHKE, 2016), Maria \& Vitorino Scale (Escala M\&V) (MARIA; VICTORINO, 1997); Digestive Disease Week Japan Scale (DDW-J) (HANATANI et al., 2014). Autores apontam o algoritmo Rucam como o padrão a ser seguido na identificação de Dili por sua sensibilidade e especificidade elevadas (DANAN; TESCHKE, 2019).

O algoritmo Rucam foi desenvolvido e aplicado originalmente na França, razão pela qual foi inicialmente chamado de "Método Francês de diagnóstico de Dili" (DANAN; TESCHKE, 2016). O instrumento tomou representatividade quando reconhecido e publicado pelo Conselho para as Organizações Internacionais de Ciências Médicas (Cioms - Councils for International Organizations of Medical Science) em 1993 (DANAN; TESCHKE, 2019). O algoritmo Rucam é largamente utilizado e existem algumas adaptações do mesmo para outras línguas (WOO et al., 2015), e, atualmente, não há versão traduzida para a língua Portuguesa (BECKER et al., 2019a). Além disso, no Brasil este algoritmo ainda é pouco utilizado. Em uma revisão sistemática de estudos de casos de lesão hepática induzida por medicamentos no Brasil, foram identificados 27 estudos e 32 casos, e apenas 4 casos reportaram o uso de algoritmo para a avaliação da causalidade, sendo estes Naranjo $(n=1)$, Maria \& Vitorino $(n=2)$ e Rucam ( $n=1)$ (BECKER et al., 2019a).

Dessa maneira, o objetivo deste trabalho é traduzir o algoritmo Rucam para o português e validar o mesmo para a utilização na prática clínica para a identificação da causalidade de lesão hepática induzida por medicamentos.

\section{MÉTODO}

A metodologia utilizada para a tradução e validação do algoritmo foi baseada no método proposto por Guillemin (1995), fundamentado nas etapas que serão descritas a seguir.

\section{Tradução}

O algoritmo Rucam foi traduzido do inglês para o português por dois profissionais cientes da finalidade do estudo - um médico e um farmacêutico. As duas traduções foram comparadas e unificadas por consenso, dando origem à versão 1 . 
A versão 1 foi traduzida (tradução inversa) do português para o Inglês por dois profissionais não cientes da finalidade. Estas traduções foram comparadas com o algoritmo original e as discrepâncias corrigidas para a versão final do questionário traduzido.

Não foi realizada adaptação cultural do instrumento traduzido, pois não existem variações culturais significativas entre os termos médicos utilizados no algoritmo; esses termos são conhecidos pelas diferentes classes profissionais incluídas na fase de validação de conteúdo do algoritmo.

\section{Validação de conteúdo}

A validação de conteúdo foi verificada pela aplicação do algoritmo traduzido, por um grupo de profissionais da saúde (médicos, farmacêuticos e enfermeiros), a um caso de suspeita de Dili.

O caso é real e foi selecionado pelos autores, sendo mantida em sigilo a identidade do paciente, conforme Quadro 1, de modo que a aplicação do algoritmo Rucam deve resultar em uma relação de causalidade altamente provável entre o uso de atorvastatina e a lesão hepática.

\footnotetext{
Paciente, sexo feminino, 65 anos de idade.

Suspeita de lesão hepática induzida por medicamento por atorvastatina $80 \mathrm{mg}$, $1 \mathrm{xdia}$.

Em uso do medicamento por 123 dias.

Sem história prévia de doenças hepáticas.

Nega consumo de álcool.

Sem história imediata de insuficiência cardíaca, choque ou sepse.

Uso de outros medicamentos concomitantes: metoclopramida (obs.: possui relato de hepatotoxicidade descrito em bula).

Exames laboratoriais na admissão.

ALT 658 U/L (12,7 vezes Limite Superior da Normalidade (LSN)).

AST $666 \mathrm{U} / \mathrm{L}(18,5$ vezes o $\mathrm{LSN})$.

Fosfatase Alcalina $861 \mathrm{U} / \mathrm{L}(6,8$ vezes $\mathrm{LSN})$.

Bilirrubina total de $0,4 \mathrm{mg} / \mathrm{dL}$.

Albumina $4,2 \mathrm{~g} / \mathrm{dL}$.

A paciente apresentou regressão dos sinais e sintomas quando o medicamento atorvastatina foi suspenso, chegando a valores normais das provas hepáticas em 45 dias.
}

A abordagem aos profissionais (médicos, farmacêuticos e enfermeiros) ocorreu por meio de questionário on-line, via plataforma survey monkey. A amostragem deu-se por conveniência, em que foram convidados, via e-mail, os alunos do Programa de Pós-Graduação em Hepatologia da Universidade Federal de Ciências da Saúde de Porto Alegre (UFCSPA), os alunos do Programa de Pós-graduação em Enfermagem da UFCSPA e profissionais que atuam em hospitais da região metropolitana de Porto Alegre, diretamente na assistência aos pacientes, vinculados ao Comitê Técnico de Farmácia do Sindicato dos Hospitais e Clínicas de Porto Alegre (Sindihospa), ou aos Hospitais Divina Providência ou Grupo Hospitalar Conceição.

Ao escore obtido ao final da aplicação do algoritmo Rucam pelos profissionais, foi atribuída uma relação de causalidade, conforme apresentado no Quadro 1.

O cálculo amostral resultou na necessidade de 41 respostas válidas ao questionário para um nível de confiança de $90 \%$ do estudo.

Os registros foram organizados em um banco de dados com as distintas classificações (variáveis) e analisados por meio do software de análises estatísticas SPSS Statistics, versão 23.

\section{Aspectos éticos e legais}

A tradução e validação do algoritmo Rucam foi autorizada previamente pelo autor principal do algoritmo.

Conforme os princípios éticos norteadores de pesquisas envolvendo seres humanos, o projeto de pesquisa foi aprovado pelo Comitê de Ética da Universidade Federal de Ciências da Saúde de Porto Alegre (UFCSPA) no 566216.0.0.0000.5345.

\section{RESULTADOS}

Durante as etapas de tradução do algoritmo foram identificadas algumas divergências que foram resolvidas por consenso entre os profissionais envolvidos. A versão final do algoritmo traduzido para o português e a escala de pontos encontram-se nos Quadro 1 e 2 . 
Quadro 1 - Algoritmo para identificação de lesão hepática induzida por plantas e medicamentos

\begin{tabular}{|c|c|c|c|}
\hline & $\begin{array}{l}\text { LESÃO HEPÁTICA DO TIPO HEPATOCELULAR } \\
\text { (ALT/FA } \geq 5)\end{array}$ & Escore & $\begin{array}{l}\text { Resultado } \\
\text { (assinalar) }\end{array}$ \\
\hline 1 & $\begin{array}{l}\text { Tempo entre a administração do medicamento ou fitoterápico e o aparecimento dos sinais e sintomas } \\
5 \text { a } 90 \text { dias (reexposição: } 1 \text { a } 15 \text { dias); } \\
\text { < } 5 \text { ou > } 90 \text { dias (reexposição: }>15 \text { dias); } \\
\text { Alternativa: tempo para início dos sinais e sintomas partindo da cessação do medicamento ou fitoterápico } \\
\leq 15 \text { dias (exceção: para metabolizadores lentos > } 15 \text { dias). }\end{array}$ & $\begin{array}{l}+2 \\
+1\end{array}$ & ( ) \\
\hline 2 & $\begin{array}{l}\text { Curso da ALT (alanina aminotransferase) após a cessação do medicamento ou fitoterápico. Diferença percentual } \\
\text { entre o pico de ALT e o limite superior da normalidade (LSN). } \\
\text { - Redução } \geq 50 \% \text { em } 8 \text { dias; } \\
\text { - Redução } \geq 50 \% \text { em } 30 \text { dias; } \\
\text { - Sem informação ou medicamento ou fitoterápico segue em uso. } \\
\text { - Redução } \geq 50 \% \text { após } 30 \text { dias; } \\
\text { - Redução }<50 \% \text { após } 30 \text { dias ou aumento recorrente da ALT } \\
\end{array}$ & $\begin{array}{c}+3 \\
+2 \\
0 \\
0 \\
-2\end{array}$ & $\begin{array}{ll}( & \\
( & \\
( & \\
( & \\
( & \\
( & \\
\end{array}$ \\
\hline 3 & $\begin{array}{l}\text { Fatores de risco: } \\
\text { - Uso de álcool (consumo de doses/dia: }>2 \text { para mulheres, }>3 \text { para homens); } \\
\text { - Uso de álcool (consumo de doses/dia: } \leq 2 \text { para mulheres, } \leq 3 \text { para homens); } \\
\text { - Idade } \geq 55 \text { anos; } \\
\text { - Idade }<55 \text { anos. }\end{array}$ & $\begin{array}{c}+1 \\
0 \\
+1 \\
0\end{array}$ & $\begin{array}{l}(1) \\
(1) \\
(1) \\
(1)\end{array}$ \\
\hline 4 & $\begin{array}{l}\text { Uso concomitante de outros medicamentos ou fitoterápicos } \\
\text { - Não utiliza medicamento ou fitoterápico concomitantemente. } \\
\text { - Medicamento ou fitoterápico em uso concomitante, porém com tempo para início dos sinais e sintomas } \\
\text { incompatível com a lesão hepática apresentada. } \\
\text { - Medicamento ou fitoterápico em uso concomitante, com tempo para início dos sinais e sintomas compatível } \\
\text { - Medicamento ou fitoterápico em uso concomitante, com conhecido efeito hepatotóxico e com tempo para início } \\
\text { dos sinais e sintomas compatível com a lesão hepática apresentada (se afirmativo, excluir alternativa anterior); } \\
\text { - Medicamento ou fitoterápico em uso concomitante, com evidencia de "causalidade" (reexposição positiva ao } \\
\text { medicamento ou fitoterápico neste caso, e alteração das transaminases). }\end{array}$ & $\begin{array}{l}0 \\
0 \\
-1 \\
-2\end{array}$ & $\begin{array}{l}\left(\begin{array}{l}1 \\
(\end{array}\right) \\
(1) \\
(\quad)\end{array}$ \\
\hline 5 & 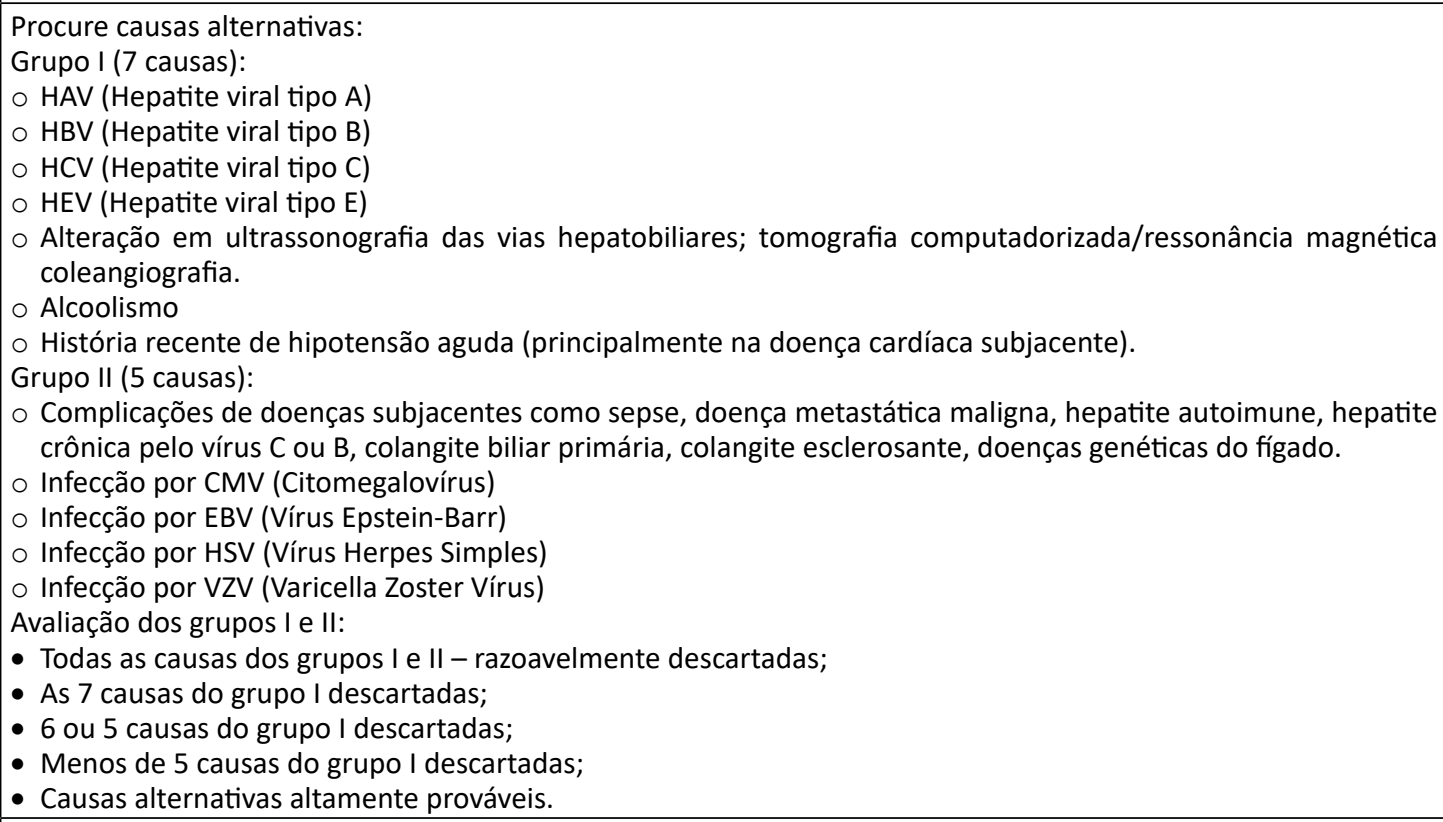 & $\begin{array}{c}+2 \\
+1 \\
0 \\
-2 \\
-3\end{array}$ & $\left.\begin{array}{l}1 \\
( \\
( \\
( \\
(\end{array}\right)$ \\
\hline 6 & $\begin{array}{l}\text { Hepatotoxicidade previamente conhecida para o medicamento ou fitoterápico suspeito } \\
\text { - É uma reação prevista em bula/rótulo para este medicamento ou fitoterápico. } \\
\text { - É uma reação publicada para este medicamento ou fitoterápico, porém não consta na bula/rótulo. } \\
\text { - É uma reação desconhecida para este medicamento ou fitoterápico. }\end{array}$ & $\begin{array}{c}+2 \\
+1 \\
0\end{array}$ & \\
\hline 7 & $\begin{array}{l}\text { Resposta a reexposição ao medicamento ou fitoterápico suspeito. } \\
\text { - Duplicação de níveis de ALT, com o medicamento ou fitoterápico isolado, posto que, anteriormente à } \\
\text { reexposição, os valores de ALT estavam abaixo de } 5 x \text { LSN. } \\
\text { - Duplicação de níveis de ALT, com o medicamento ou fitoterápico administrado nas mesmas condições da } \\
\text { primeira reação. } \\
\text { - Aumento da ALT, porém ainda abaixo do LSN nas mesmas condições da primeira administração. } \\
\text { - Outras situações. }\end{array}$ & $\begin{array}{c}+3 \\
+1 \\
-2 \\
0\end{array}$ & $\begin{array}{l}(1) \\
(1)\end{array}$ \\
\hline
\end{tabular}

Fonte: Traduzido e elaborado pelos autores. 
Quadro 2 - Relação entre os possíveis valores obtidos pela aplicação do algoritmo Rucam e a causalidade para lesão hepática induzida por medicamentos

\begin{tabular}{|l|c|}
\hline Escore & Classificação da causalidade \\
\hline$\leq 0$ & Relação excluída \\
\hline 1 a 2 & Relação improvável \\
\hline 3 a 5 & Relação possível \\
\hline 6 a 8 & Relação provável \\
\hline$\geq 9$ & Relação altamente provável \\
\hline
\end{tabular}

Fonte: Versão original: DANAN; TESCHKE, 2016. Traduzido pelos autores.

O link de acesso ao algoritmo foi encaminhado para 168 profissionais. Destes, 53 profissionais responderam, e quatro respostas foram excluídas por não contemplarem as categorias profissionais objeto deste estudo (nutricionistas e biomédicos). Oito respostas foram excluídas por não estarem completas, resultando em um total de 41 respostas, sendo Enfermeiros ( $n=16)$, Farmacêuticos $(n=19)$ e Médicos $(n=6)$. O tempo médio observado para a resposta ao questionário foi de 17 minutos com desvio padrão de 10 minutos.

O escore médio de causalidade de acordo com o algoritmo Rucam foi de 7,58 $\pm 3,48$, o que denota a classificação de causalidade como provável para o caso clínico avaliado. O escore esperado para a aplicação do algoritmo ao caso clínico apresentado era de 9, condizente com a classificação de altamente provável. Este resultado foi observado em 18 (44\%) das respostas válidas dos profissionais, sendo Enfermeiros $(n=6)$, Farmacêuticos $(n=10)$ e Médicos $(n=2)$.

A frequência da causalidade, de acordo com a categoria profissional, pode ser visualizada na Tabela 1 . As diferenças encontradas entre os resulta- dos para as diversas categorias profissionais não foram estatisticamente significativas (Krus Kall Wallis $p=0,800)$.

Se observadas as perguntas isoladamente, o percentual de acertos em relação à resposta esperada foi inferior a $80 \%$ em quatro dos oito questionamentos, conforme demonstrado na Tabela 2. As perguntas relacionadas ao consumo prévio de álcool, ao uso concomitante de outro medicamento com potencial hepatotóxico, a ocorrência de causas alternativas que justifiquem a condição do paciente (sepse, doença metastática maligna, hepatite autoimune, hepatite crônica pelo vírus $\mathrm{C}$ ou $\mathrm{B}$, colangite biliar primária, colangite esclerosante, doenças genéticas do fígado, alcoolismo, infecções virais - citomegalovírus, Epstein-Barr, Herpes Simples, Varicela) e a resposta à reexposição ao medicamento suspeito, obtiveram maior variabilidade de respostas entre as diferentes categorias profissionais.

\section{DISCUSSÃO}

Embora tenhamos atingido o número de participantes para a validação da tradução, a adesão dos profissionais à participação na pesquisa foi considerada baixa. Apenas $24 \%$ dos profissionais convidados realizaram a aplicação do algoritmo. O fato foi atribuído primeiramente à metodologia utilizada. Uma das limitações da utilização de questionários eletrônicos é o baixo índice de respostas. Além disso, atribuiu-se ao possível desinteresse ou desconhecimento destes profissionais sobre o tema, apesar de que todos os convidados atuam na assistência ao paciente. Outro fator importante é que os hospitais onde os profissionais atuam não possuem comissão de farmacovigilância ativa.

Tabela 1 - Classificação da causalidade da lesão

hepática induzida por medicamentos de acordo

com o algoritmo Rucam e distribuídos por categoria profissional

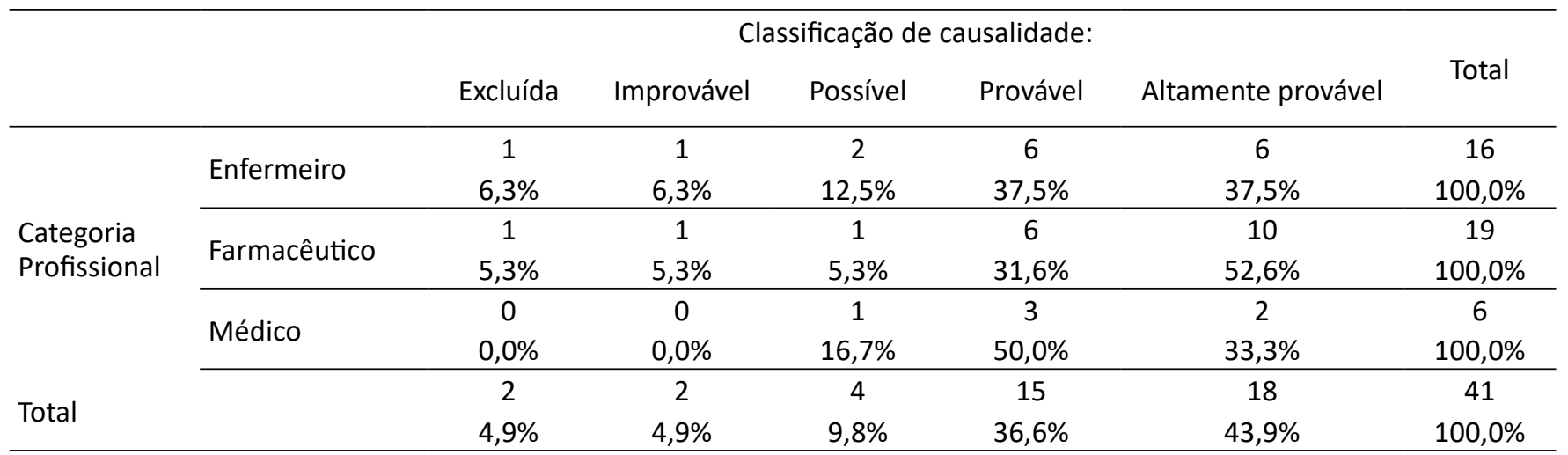

$p=0,957$ Pearson Chi-square. 
Tabela 2 - Número e percentual de acertos por categoria profissional para as perguntas do algoritmo Rucam

\begin{tabular}{|c|c|c|c|c|c|}
\hline \multirow[b]{2}{*}{ Temática da pergunta } & \multicolumn{5}{|c|}{ Número de acertos } \\
\hline & $\begin{array}{c}\text { Enfermeiros } \\
(n=16)\end{array}$ & $\begin{array}{c}\text { Farmacêuticos } \\
(n=19)\end{array}$ & $\begin{array}{l}\text { Médicos } \\
(\mathrm{n}=06)\end{array}$ & $\begin{array}{l}\text { Todos } \\
(\mathrm{n}=41)\end{array}$ & $\begin{array}{c}\text { Todos } \\
(\%)\end{array}$ \\
\hline $\begin{array}{l}\text { Relação temporal entre administração do } \\
\text { medicamento e o aparecimento dos sinais e } \\
\text { sintomas }\end{array}$ & 12 & 16 & 5 & 33 & 80,52 \\
\hline Curso de ALT após a retirada do medicamento & 12 & 17 & 5 & 34 & 82,96 \\
\hline Consumo de álcool & 10 & 16 & 5 & 31 & 75,64 \\
\hline Idade & 15 & 19 & 6 & 40 & 97,60 \\
\hline $\begin{array}{l}\text { Uso concomitante de outro medicamento } \\
\text { hepatotóxico }\end{array}$ & 7 & 7 & 2 & 16 & 39,04 \\
\hline $\begin{array}{l}\text { Ocorrência de causas alternativas que justifiquem } \\
\text { a condição do paciente }\end{array}$ & 8 & 14 & 3 & 25 & 61,00 \\
\hline $\begin{array}{l}\text { Hepatotoxicidade previamente conhecida para o } \\
\text { medicamento suspeito }\end{array}$ & 12 & 16 & 6 & 34 & 82,96 \\
\hline Resposta à reexposição ao medicamento suspeito & 8 & 10 & 4 & 22 & 53,68 \\
\hline
\end{tabular}

Fonte: Elaborada pelos autores.

Os escores resultantes da aplicação do algoritmo Rucam ao caso clínico em estudo por diferentes profissionais, é estatisticamente igual. Com base nesta informação, podemos considerar a tradução do algoritmo para a língua portuguesa como validada.

Em uma revisão sistemática dos casos de Dili no Brasil, 27 estudos reportaram 32 casos. A maior parte dos casos foi identificada em hospitais, e ocorreu principalmente em homens jovens com doenças crônicas. Foram relatadas 7 mortes e 2 casos de transplante hepático, o que demonstra a gravidade do problema, e cuja detecção precoce pode mudar o percurso da lesão hepática, uma vez que em $50 \%$ dos casos em que houve a suspensão do medicamento ocorreu a normalização das enzimas hepáticas (BECKER et al., 2019a).

Segundo Danan e Teschke (2016), autores do algoritmo Rucam, este pode ser utilizado pelas diferentes categorias profissionais da área da saúde. Estudo apresenta que o instrumento pode ser aplicado por profissionais sem a necessidade de que sejam especialistas na temática da lesão hepática induzida por medicamentos (DEVARBHAVI, 2012).

O caso clínico escolhido para a aplicação do algoritmo foi cuidadosamente descrito como sugere a literatura, contendo a história do paciente, a evolução dos sinais e sintomas, os exames realizados, o diagnóstico médico e o tratamento insituído, de modo a minimizar possíveis dúvidas de interpretação.
O tempo médio para a resposta ao questionário foi elevado ( $17 \mathrm{~min} \pm 10 \mathrm{~min}$ ), se comparado ao tempo estimado pelos autores para sua aplicação ( 5 minutos). Atribuiu-se a este resultado a complexidade do algoritmo em si, e ao fato de ser o primeiro contato dos respondentes com o instrumento, embora Devarbhavi (2012) afirme que o questionário pode ser aplicado por não especialistas. Para os profissionais mais familiarizados com o assunto, o algoritmo possui maior reprodutibilidade e é possível reduzir o tempo gasto para a sua utilização (GARCÍA-CORTÉS et al., 2011). Dessa maneira, sugere-se a educação continuada da equipe para aplicação do questionário.

O algoritmo Rucam possui oito questões - decorrentes de sete domínios: tempo de início da doença hepática, duração da doença, fatores de risco, uso concomitante de medicamentos, exclusão de causas alternativas, história prévia do medicamento suspeito e resposta à reexposição ao medicamento (DANAN; TESCHKE, 2016) - porém, para algumas, as alternativas possuem textos longos e complexos, o que pode requerer maior tempo para interpretação.

O escore médio observado entre as diferentes categorias profissionais $(7,58 \pm 3,48)$ foi inferior ao esperado (9 pontos), e levou à classificação do caso como provável para a relação de causalidade entre a condição do paciente e o uso de atorvastatina. Avaliando-se individualmente as questões, é possível identificar aquelas em que a divergência entre as respostas foi maior. A pergunta que avalia o consumo de álcool, por exemplo, obteve um percentual de erro de 
24\%. Tratava-se de uma pergunta simples, objetiva, com apenas duas respostas possíveis. Atribui-se a este resultado a dificuldade individual dos profissionais na interpretação.

O álcool é considerado um fator de risco pelo algoritmo Rucam. Não é, porém, por si só, conhecido por aumentar o risco de desenvolvimento idiossincrático de Dili. Além disso, a evidência de que a ingestão crônica de álcool aumenta a susceptibilidade de Dili é baseada em achados com número limitado de medicamentos, e pode não ser universalmente aplicável (CHALASANI et al., 2008; GARCÍA-CORTÉS et al., 2011).

Um índice de erros elevado foi também verificado para o uso concomitante de outros medicamentos ou fitoterápicos e relação quanto ao seu potencial hepatotóxico. O caso clínico descrevia o uso concomitante de três medicamentos. Para cada um destes foi descrito as reações adversas constantes em bula, incluindo a hepatotoxicidade para um dos medicamentos citados. Ainda assim, houve grande divergência entre as respostas. Mais uma vez atribuiu-se a divergência às dificuldades individuais de interpretação, bem como à desatenta leitura do caso clínico, ou ao fato da não importância do relato de hepatotoxicidade em bula. Como inúmeros fatores precisam ser avaliados para a aplicação do algoritmo, é necessário que os aplicadores tenham familiaridade com o mesmo, o que talvez não pode ser adquirido em uma única aplicação.

Em relação ao questionamento quanto à busca de causas alternativas à condição do paciente, o percentual de acertos foi de $61 \%$. A exclusão de outras causas potenciais de lesão hepática é essencial na avaliação da causalidade. A frequência de doenças específicas do fígado varia de acordo com as populações e localização geográfica. Em geral, a frequência de causas não relacionadas com fármacos é, muitas vezes, consideravelmente mais elevada do que a Dili (WOO et al., 2015; TESCHKE, 2019). As causas alternativas incluem doenças hepáticas e distúrbios extra-hepáticos com comprometimento do fígado, tais como hepatites virais, hepatite autoimune, citomegalovírus, Epstein-Barr vírus, colelitíase, obstruções biliares, isquemia hepática e hipóxia por insuficiência cardíaca ou circulatória, sepse sistêmica e síndrome de Gilbert (DANAN; TESCHKE, 2016). A etapa de exclusão de causas alternativas à condição dos pacientes é a etapa mais complexa e abrangente da aplicação do algoritmo Rucam (DANAN; TESCHKE, 2019; TESCHKE, 2019).

A idade avançada - mais de 55 anos - é um dos fatores de risco avaliados pelo Rucam. Dados recentes de uma grande coorte, no entanto, indicam que a ida- de mais avançada não predispõe à ocorrência de Dili, mas pode ser um preditor de dano colestático, enquanto a idade mais jovem parece estar relacionada ao padrão de lesão hepatocelular (GARCÍA-CORTÉS et al., 2011; TESCHKE; ANDRADE, 2016; TESCHKE, 2019).

Atualmente, a maioria dos especialistas concorda que as variações genéticas são provavelmente o maior fator de risco para Dili (GARCÍA-CORTÉS et al., 2011; DANJUMA et al., 2019). Neste contexto, o desenvolvimento de biomarcadores poderá contribuir de forma ainda mais objetiva e assertiva para o diagnóstico de doença hepática induzida por medicamentos (DANJUMA et al., 2019).

Em relação aos testes clínicos para a investigação da DILI, normalmente apenas os padrões bioquímicos são utilizados no diagnóstico. Isto porque a análise histopatológica é muito invasiva, e os achados não serão, em um primeiro momento, determinantes para a definição do tratamento que normalmente parte da interrupção deste com o medicamento suspeito (TESCHKE; FRENZEL, 2014; LICATA, 2016). O papel da biópsia hepática no diagnóstico Dili é uma questão controversa. Para alguns, a biópsia deve ser realizada quando o paciente pode ter uma lesão hepática subjacente ou caracterizar padrões de lesão desconhecidos, levando a lesões mais graves ou residuais que possam ter significância prognóstica (GARCÍA-CORTÉS et al., 2011; HASSAN; FONTANA, 2019). Por outro lado, também apresenta-se que as características histopatológicas do fígado observadas nos casos de Dili são semelhantes às características das outras doenças hepáticas e biliares primárias, não havendo alterações específicas para o Dili, o que não justificaria a realização de biópsia hepática (TESCHKE; FRENZEL, 2014).

$O$ relato de hepatotoxicidade prévia dos medicamentos é um fator contribuinte na determinação do escore no Rucam. Esta, contudo, só será reproduzível se houver uma fonte de dados comum com atualização contínua da informação, ou o julgamento será amplamente dependente do estado de informação de cada avaliador. O significado de "potencial de hepatotoxicidade" é, portanto, deixado ao avaliador para interpretar, inevitavelmente levando a variações entre avaliadores. No que se refere aos fitoterápicos, esta informação torna-se ainda mais difícil de ser acessada pela falta de publicações e uniformidade de conceitos entre elas (GARCÍA-CORTÉS et al., 2011; TESCHKE; ANDRADE, 2016; BYEON et al., 2019).

Indiscutivelmente, a reesposição do paciente ao fármaco suspeito com reaparecimento dos sinais e sintomas (reexposição positiva), é o fator mais consistente da determinação do diagnóstico de Dili (DANAN; 
TESCHKE, 2016). Esta prática, porém, é contraindicada e raramente realizada em razão dos riscos envolvidos. Embora situações de reexposição ao medicamento sejam raras, quando ocorrem podem ter respostas altamente variáveis e retardadas à readministração, podendo levar a suposições errôneas (GARCÍA-CORTÉS et al., 2011; STINE; CHALASANI, 2015).

Entre as diferentes categorias profissionais, os farmacêuticos demonstraram maior aproximação ao resultado esperado. Tal situação pode estar relacionada a uma característica da profissão, e por possuir maior facilidade com a utilização de algoritmos ou mais familiarizado com a prática da Farmacovigilância, atuando diariamente na identificação e classificação de RAM. Tal resultado corrobora a necessidade de treinamento de médicos, enfermeiros e farmacêuticos para a investigação da causalidade de lesão hepática induzida por medicamentos.

Estima-se que desde o início da década de 70 do século 20 mais de 3 milhões de casos de lesão hepática induzida por medicamentos já foram notificados. Destes, no entanto, apenas um terço dos casos possui algum critério de causalidade empregado para o diagnóstico (DEVARBHAVI, 2012; STINE; CHALASANI, 2015).

Estimular o uso do algoritmo pelos diferentes profissionais das equipes de saúde e a identificação precoce de Dili, é importante para a segurança do paciente. $\mathrm{O}$ uso do algoritmo confere consistência à suspeita de RAM, à medida que atribui grau de causalidade à ocorrência (TAN et al., 2018).

0 reconhecimento imediato do medicamento causador da lesão hepática é o aspecto mais importante no manejo da hepatotoxicidade, uma vez que parece diminuir o risco de progressão para insuficiência hepática aguda ou lesão hepática crônica (GARCÍA-CORTÉS et al., 2011; DEVARBHAVI, 2012). Vários aspectos do Dili dificultam seu diagnóstico, principalmente por assemelhar-se a qualquer doença hepática aguda ou crônica, e por não possuir um padrão específico (manifestações clínicas e patológicas) por medicamento (GARCÍA-CORTÉS et al., 2011; DANAN; TESCHKE, 2019).

$O$ algoritmo Rucam é largamente utilizado para a identificação de Dili, sendo preferido por alguns autores em relação aos algoritmos de Naranjo (Naranjo Adverse Drug Reactions Probability Scale - NADRPS), Digestive Disease Week Japan (DDW-J), Maria \& Victorino $(M \& V)$ e Drug induced liver injury networking (Dilin). A preferência decorre de sua sensibilidade e especificidade para identificação de lesão hepática induzida por medicamentos, e de resultados de estu- dos comparativos nos quais se mostrou mais confiável e com resultados mais reprodutíveis (DEVARBHAVI, 2012; DANAN; TESCHKE, 2019).

Autores apontam algumas limitações do algoritmo Rucam no que refere à complexidade dos termos empregados, o que decorre, na maioria das vezes, em maior tempo para sua aplicação aos casos clínicos. Para profissionais que não possuem maior familiaridade com a rotina de identificação de Dili, a aplicação do algoritmo requer maior dedicação para a compreensão e utilização (GARCÍA-CORTÉS et al., 2011). Partindo deste princípio, a tradução do questionário para a língua Portuguesa favorece a utilização e a disseminação do algoritmo entre os profissionais das equipes de saúde do Brasil, na medida em que facilita o entendimento dos aspectos abordados.

Outra limitação do algoritmo, que na verdade é uma limitação de todos os sistemas de pontuação para determinação de causalidade, é de que este não confere certeza ao diagnóstico. O sistema de pontuação do algoritmo Rucam infere, no máximo, a uma relação de causalidade altamente provável. Apesar disto, muitos estudos utilizam o algoritmo Rucam para a determinação do diagnóstico de Dili (HANATANI et al., 2014; DANAN; TESCHKE, 2019; TESCHKE, 2019).

A utilização dos algoritmos para a identificação da doença hepática induzida por medicamentos reduz a subjetividade do diagnóstico, tornando mais consistente a decisão da equipe multidisciplinar no seguimento terapêutico do paciente (TAN et al., 2018; MILJKOVIC; DOBRIC; DRAGOJEVIC-SIMIC, 2012; DEVARBHAVI, 2012; GARCÍA-CORTÉS et al., 2011). Alguns autores, contudo, afirmam que não é necessária a confirmação e/ou a complementação dessa avaliação pelo especialista (GARCÍA-CORTÉS et al., 2011).

Nos casos em que há falta de informações acerca da condição e histórico do paciente, o escore sofre uma redução de probabilidade, o que, de modo geral, não reduz, na verdade, a possibilidade de que não seja decorrente do medicamento a sua condição. Da mesma forma, para os casos em que há um período de latência superior ao usualmente observado - quer pelas condições farmacogenéticas do paciente ou por outras situações de atenuação dos sinais e sintomas o algoritmo atribui resultados que não condizem com a real relação do medicamento à lesão hepática do paciente (GARCÍA-CORTÉS et al., 2011; LICATA, 2016).

Outra limitação do algoritmo está na forma de atribuição de valores - pontuação - aos domínios das perguntas, podendo, muitas vezes, o resultado subestimar a causalidade. Como exemplo, o autor cita os casos em que há avaliação de múltiplos medicamen- 
tos (nas associações medicamentosas em uma mesma a apresentação) nos critérios da idade, e nos casos em que há reexposição ao medicamento (DEVARBHAVI, 2012).

Entre as vantagens do algoritmo Rucam, a principal está em proporcionar reprodutibilidade entre as avaliações de diferentes avaliadores para os casos em que há suspeita de Dili (HANATANI et al., 2014; DANAN; TESCHKE, 2019; TESCHKE, 2019). A utilização do algoritmo de Rucam confere maior objetividade à avaliação e possibilita avaliar a força da probabilidade de uma determinada condição (excluída, improvável, possível, provável, altamente provável).

O algoritmo de Rucam foi desenvolvido não apenas para sua utilização em medicamentos alopáticos, mas também para a verificação de nexo causal entre a lesão hepática e a utilização de fitoterápicos (DANAN; TESCHKE, 2019). No Brasil, é muito disseminada a cultura de que "o natural não faz mal", razão pela qual os fitoterápicos são amplamente consumidos, inclusive na sua forma "in natura". Além disso, a população muitas vezes não identifica o fitoterápico como um medicamento. Apesar disso, há relatos de lesão hepática induzida por plantas e fitoterápicos. Recentemente nosso grupo publicou um estudo de uso de Kava Kava por tempo inferior a 60 dias que levou à lesão hepática e necessidade de transplante hepático (BECKER et al., 2019b). Evidenciando a importância do algoritmo Rucam para a população brasileira e investigação da suspeite de Dili com o uso de medicamentos e fitoterápicos.

\section{CONCLUSÃO}

O algoritmo Rucam traduzido para a língua portuguesa que verifica a causalidade de lesão hepática e os medicamentos, é uma importante ferramenta para a prática clínica e pode ser aplicado por médicos, farmacêuticos e enfermeiros. A aplicação do algoritmo permite maior acurária na confirmação da suspeita da reação adversa, além de contribuir para a saúde pública, à medida que favorece a identificação dos medicamentos com potencial hepatotóxico na fase de comercialização, permitindo a notificação e ações de retirada do mercado de medicamentos com baixa segurança.

\section{REFERÊNCIAS}

BECKER, M. W. et al. Drug and herb-induced liver injury: a critical review of Brazilian cases with proposals for the improvement of causality assessment using RUCAM.Annals of Hepatology, v. 18, n. 5, p. 742-750, 2019a.
BECKER, M. W. et al. Liver transplantation and the use of KAVA: Case report. Phytomedicine, v. 56, p. 21-26, 2019b.

BLATT, C.;. BECKER, M. W.; LUNARDELLI, M. J. M. Lesão hepática induzida por medicamentos: qual o papel do farmacêutico clínico. Revista Brasileira de Farmácia Hospitalar e Serviços de Saúde, v. 7, n. 4, 2016.

BYEON, J-H. et al. Systematic review of published data on herb induced liver injury. Journal of ethnopharmacology, v. 233, p. 190-196, 2019.

CHALASANI, N. et al. Causes, clinical features, and outcomes from a prospective study of drug-induced liver injury in the United States. Gastroenterology, v. 135, n. 6, p. 1.9241.934, 2008.

DANAN, G.; TESCHKE, R. RUCAM in drug and herb induced liver injury: the update. International Journal of Molecular Sciences, v. 17, n. 1, p. 14, 2016.

DANAN, G.; TESCHKE, R. Roussel Uclaf Causality Assessment Method for drug-induced liver injury: present and future. Frontiers in Pharmacology, v. 10, 2019.

DANJUMA, M. I-M. et al. Novel biomarkers for potential risk stratification of drug induced liver injury (DILI): A narrative perspective on current trends. Medicine, v. 98, n. 50, p. e18322, 2019.

DEVARBHAVI, H. An update on drug-induced liver injury. Journal of Clinical and Experimental Hepatology, v. 2, n. 3, p. 247-259, 2012.

GARCÍA-CORTÉS, M. et al. Spanish Group for the Study of Drug-Induced Liver Disease (Grupo de Estudio para las Hepatopatías Asociadas a Medicamentos GEHAM). Causality assessment methods in drug induced liver injury: strengths and weaknesses. J. Hepatol., v. 55, n. 3, p. 683-691, 2011.

GUILLEMIN, F. Cross-cultural adaptation and validation of heatth status measures. Scandinavian Journal of Rheumatology, v. 24, n. 2, p. 61-63, 1995.

HANATANI, T. et al. A detection algorithm for drug-induced liver injury in medical information databases using the Japanese diagnostic scale and its comparison with the council for international organizations of medical sciences/the roussel uclaf causality assessment method scale. Pharmacoepidemiology and Drug Safety, v. 23, n. 9, p. 984-988, 2014.

HASSAN, A.; FONTANA, R. J. The diagnosis and management of idiosyncratic drug-induced liver injury. Liver International, v. 39, n. 1, p. 31-41, 2019.

LICATA, A. Adverse drug reactions and organ damage: the liver. European Journal of Internal Medicine, v. 28, p. 9-16, 2016.

MARIA, V. A.; VICTORINO, R. M. Development and validation of a clinical scale for the diagnosis of drug-induced hepatitis. Hepatology, v. 26, n. 3, p. 664-669, 1997.

MILJKOVIC, M. M.; DOBRIC, S.; DRAGOJEVIC-SIMIC, V. ACcuracy and reproducibility of two scales in causality assessment of unexpected hepatotoxicity. Journal of Clinical Pharmacy and Therapeutics, v. 37, n. 2, p. 196-203, 2012.

NARANJO, C. A. et al. A method for estimating the probability of adverse drug reactions. Clinical Pharmacology and Therapeutics, 30, p. 239-45, 1981. 
STINE, J G.; CHALASANI, N. Chronic liver injury induced by drugs: a systematic review. Liver International, v. 35, n. 11, p. 2.343-2.353, 2015.

TAN, E H et al. Systematic review and meta-analysis of algorithms used to identify drug-induced liver injury (DILI) in health record databases. Liver International, v. 38, n. 4, p. 742-753, 2018.

TESCHKE, R. Idiosyncratic DILI: Analysis of 46,266 cases assessed for causality by RUCAM and published from 2014 to early 2019. Frontiers in pharmacology, v. 10, 2019.

TESCHKE, R.; ANDRADE, R. J. Drug, herb, and dietary supplement hepatotoxicity. Int. J. Mol. Sci., Sept, v. 17, n. 9, p. 1.488, 2016.

TESCHKE, R.; FRENZEL, C. Drug induced liver injury: do we still need a routine liver biopsy for diagnosis today? Annals of Hepatology, v. 13, n. 1, p. 121-126, 2014.

WOO, H. J. et al. Drug-induced liver injury: A 2-year retrospective study of 1169 hospitalized patients in a single medical center. Phytomedicine, v. 22, n. 13, p. 1.201-1.205, 2015. 